\title{
ON APPROXIMATE DERIVATIVES ${ }^{1}$
}

CHOW, SHU-ER

1. Introduction and definitions. In the present paper $f(x)$ will signify a real, one-valued function defined in the interval $I:(a, b)$. All sets we shall have occasion to speak of will be subsets of $I$.

The theorem of Denjoy on Dini derivatives, as extended by S. Saks $[1,(1)],{ }^{2}$ H. E. Hanson [2], and H. Blumberg [3] to arbitrary functions $f(x)$, is briefly as follows: At almost every point $x$ we have either $(\alpha)$ the derivative of $f(x)$ exists and is finite-we shall then say that the directional angle of the curve $y=f(x)$ is $0^{\circ}$ at $x$; or $(\beta)$ two opposite derivatives (the lower Dini derivative on one side and the upper Dini derivative on the other side) are finite and equal, and the other two are $\pm \infty$ respectively - we then say that the directional angle is $180^{\circ}$ at $x$; or $(\gamma)$ the upper Dini derivatives are $+\infty$ and the lower $-\infty$ -we then say that the directional angle is $360^{\circ}$ at $x$.

Another line of generalization is concerned with the use of approximate derivatives, which we proceed to define.

Definition 1. The upper right approximate limit of $f(x)$ at $\xi-$ which we denote by $u^{+}(f, \xi)$, or simply by $u^{+}(\xi)$ when there is no ambiguity -is the g.l.b. of the (real) numbers $k$ for which the set

$$
E_{x}[f(x)>k, x>\xi]
$$

has zero exterior (metric) density at $\xi$. Similarly, the lower right approximate limit of $f(x)$ at $\xi$-denoted by $l^{+}(f, \xi)$, or $l^{+}(\xi)$-is the l.u.b. of the numbers $k$ for which the set

$$
E_{x}[f(x)<k, x>\xi]
$$

has zero exterior density at $\xi$. The two left-hand limits, $u-(\xi)$ and $l(\xi)$, are defined similarly.

Without reference to the (left or right) direction of approach, we define the upper and lower approximate limits of $f(x)$ at $\xi$ respectively as

$$
u(\xi)=\max \left[u^{+}(\xi), u^{-}(\xi)\right], \text { and } l(\xi)=\min \left[l^{+}(\xi), l^{-}(\xi)\right] \cdot{ }^{3}
$$

Received by the editors April 1, 1947.

1 The author wishes to express his gratitude to Professor H. Blumberg, who suggested the application of "the measurable boundaries," and assisted in the editing.

${ }^{2}$ Numbers in brackets refer to the bibliography at the end of the paper.

${ }^{3}$ The functions $u(x)$ and $l(x)$ are called the "upper" and "lower metric boundaries" of $f(x)$ by Prof. Blumberg [3]. 
DeFINITION 2. The upper right approximate derivative of $f(x)$ at $\xi-$ which we shall denote by $A^{+}(f, \xi)$, or simply by $A^{+}(\xi)$-is the upper right approximate limit of the quotient function

$$
Q(f, \xi, x)=\frac{f(x)-f(\xi)}{x-\xi} .
$$

We define similarly the other three approximate derivatives, namely, the lower right $A_{+}(\xi)$, the upper left $A^{-}(\xi)$, and the lower left $A_{-}(\xi)$.

If all these four approximate derivatives are equal, their common value is called the approximate derivative of $f(x)$ at $\xi$, and denoted by $A(f, \xi)$, or $A(\xi)$. If, furthermore, $A(\xi)$ is finite, $f(x)$ is said to possess an approximate derivative, or be approximately derivable at $\xi$.

The approximate derivative, as defined, has been the basis of generalizations of Denjoy's theorem by J. C. Burkill and U. S. Haslam-Jones (among others). They proved the following result [4, (1) ] - in which it is apparent what meaning to attach to the phrase, "the directional angle with respect to approximate derivation":

If $f(x)$ is measurable, the directional angle, in respect to approximate derivation, of the curve $y=f(x)$, is almost everywhere either $0^{\circ}$ or $360^{\circ}$. Consequently, if one of the approximate derivatives of $f(x)$ is finite at every point of a set $E$, then $f(x)$ is approximately derivable at almost every point of $E$.

For approximate derivatives of non-measurable functions, there is an isolated result by A. J. Ward [5], and another by Burkill and Haslam-Jones [4, (2)]. In his Theory of the Integral, Saks [1, (2)] gives a proof of the foregoing theorem of Burkill and Haslam-Jones, and remarks that, by a "slight modification" of the proof, the theorem may be extended "in a certain way" to unconditioned functions. However, he gives no explicit proof. ${ }^{4}$ But we prove in the present paper that the Denjoy analogue for approximate derivatives for the case of non-measurable functions is different from the literature result for measurable functions.

The present paper communicates the proper analogue of Denjoy's theorem for approximate derivatives of unconditioned functions, and the proof is simple and straightforward. It turns out for this extensionin contradistinction to the corresponding result for measurable functions - that each of the cases $(\alpha),(\beta)$ and $(\gamma)$ can occur on a set of positive exterior measure. The reasoning also shows just why case $(\beta)$ drops

4 Curiously, he makes no reference to Jeffery's paper on the same question [6] which appeared prior to the publication of the book of Saks and was reviewed by him in Zentralblatt für Mathematik [7]. 
out when $f(x)$ is measurable.

In a paper by R. L. Jeffery [6], on the approximate derived numbers of arbitrary functions, the results summarized in his Theorem VI are similar to our main results. Jeffery uses an unusual definition of approximate derived number, and his work is based on the unusual idea of metric separability. His proofs are difficult to read. He does not develop the proofs of the preceding results, and omits the discussion of the existence, in his sense, of the pertinent approximate derivative numbers in case the hypotheses of his Theorems IV and $\mathrm{V}$ are not satisfied.

The principal means employed for obtaining our stated results is the theorem of Professor Blumberg on the "measurable boundaries of an arbitrary function." This theorem [3, p. 272] is as follows:

THEOREM (BLUMBERG). With every real function $f(x)$, one- or manyvalued, defined on an interval $I$, there are uniquely associated two functions $u(x)$ and $l(x)$, defined in $I$, having the following properties:

(i) $u(x)$ and $l(x)$ are measurable.

(ii) The set of points $x$ for which $f(x)>u(x)$ or $f(x)<l(x)$ is of measure zero.

(iii) The points $(x, u(x))$ and $(x, l(x))$ are positively approached ${ }^{5}$ by the curves $y=u(x), y=l(x)$, respectively, for every $x$; these points are fully approached ${ }^{6}$ by the curve $y=f(x)$ for almost every $x$, and positively approached for every $x$.

This theorem gives a structural representation of an unconditioned function $f(x)$ which shows that $f(x)$ is necessarily built-in the words of Professor Blumberg-"on the scaffolding of two measurable functions," the measurable boundaries of $f(x)$. It is this structure which permits the transfer of various theorems on measurable functions to arbitrary functions. The memoir of Professor Blumberg cites diverse interesting cases where this transfer can be effected. The present paper thus adds another application of this theorem.

2. Lemmas. In this section, we prove two lemmas which are utilized in the proof of the theorems of $\$ 3$.

LEMMA 1. For every real function $f(x)$, the set $E=E_{x}\left[u^{+}(x) \neq u^{-}(x)\right]$ is of measure zero. $A$ like result holds for $l^{+}(x)$ and $l^{-}(x)$.

6 The point $(\xi, \eta)$ is positively approached by the curve or function $y=f(x)$ if for every pair $k, l$ of real numbers, with $k<\eta<l$, the set $E_{k l}=E_{x}[k<f(x)<l]$ has positive $u p p e r$, exterior density at $\xi$.

' The point $(\xi, \eta)$ is fully approached by $y=f(x)$, if for every pair $k, l$, with $k<\eta<l$, $E_{k l}$ is of exterior density 1 at $\xi$. 
Proof. Let $E_{1}=E_{x}\left[u^{+}(x)>u^{-}(x)\right]$. For a fixed rational number $r$, let $E_{r}=E_{x}\left[u^{+}(x)>r>u^{-}(x)\right]$. Then

$$
E_{1}=\sum_{r} E_{r}
$$

where $r$ ranges over the set of rational numbers. On account of the definition of $u^{ \pm}(x)$, and the condition for the points of $E_{r}$, the set

$$
E_{x}[f(x)>r, x>\xi]
$$

has positive upper exterior density at every point $\xi$ of $E_{r}$; and the set

$$
E_{x}[f(x)>r, x<\xi]
$$

has zero exterior density at $\xi$. Hence the set

$$
E_{x}[f(x)>r]
$$

has positive upper exterior density at the right and zero exterior density at the left of every point of $E_{r}$. Now an arbitrary set has its exterior density either 0 or 1 almost everywhere. Hence $E_{r}$, and therefore $E_{1}$, is of measure zero. Similarly, the set of points $x$ for which $u^{+}(x)<u^{-}(x)$ is of measure zero. We conclude that $E$ is of measure zero.

It follows similarly that the set $E_{x}\left[l^{+}(x) \neq l-(x)\right]$ is of measure zero.

LEMma 2. For every real function $f(x)$, if $u(\xi)=f(\xi)$, then $A^{+}(u, \xi)$ $=A^{+}(f, \xi)$ and $A_{-}(u, \xi)=A_{-}(f, \xi)$. Similarly, if $l(\xi)=f(\xi)$, then $A^{-}(l, \xi)=A^{-}(f, \xi)$ and $A_{+}(l, \xi)=A_{+}(f, \xi)$.

Proof. Suppose $u(\xi)=f(\xi)$. Since $u(x) \geqq f(x)$ for almost every $x$, we have

$$
Q(u, \xi, x) \geqq Q(f, \xi, x) \quad \text { for a.e. } x>\xi \text {. }
$$

Hence

$$
A^{+}(u, \xi) \geqq A^{+}(f, \xi) .
$$

On the other hand, suppose $A^{+}(f, \xi)=k$. We may assume that $k<+\infty$; for if $k=+\infty$, we have $A^{+}(u, \xi) \geqq A^{+}(f, \xi)=+\infty$, whence $A^{+}(u, \xi)=A^{+}(f, \xi)$. For a given $\epsilon>0$, let $\lambda$ be the straight line through the point $(\xi, f(\xi))=(\xi, u(\xi))$ with slope $k+\epsilon$. Then, by the definition of $A^{+}(f, \xi)$, the set $E$ of numbers $x>\xi$ for which the point $(x, f(x))$ lies above $\lambda$ has exterior density zero at $\xi$. Hence for every $\eta>0$, there exists a positive number $h$ such that 


$$
m_{e}(I E)<\eta l
$$

for every interval $I$ with $\xi$ as left end point and length $l<h$, where $m_{e}(I E)$ denotes the exterior Lebesgue measure of $I E$.

If $\bar{E}=I-I E$, we have

$$
m_{i}(\bar{E})>(1-\eta) l
$$

where $m_{i}(\bar{E})$ denotes the interior Lebesgue measure of $\bar{E}$. Let $T$ be a measurable subset of $\bar{E}$ of measure greater than $(1-\eta) l$ and such that the density of $T$ is 1 at each of its points. For every $x$ of $\bar{E}$, the point $(x, f(x))$ lies on or below the line $\lambda$. Since, by the theorem of Blumberg, the point $(x, u(x))$ is positively approached by $y=f(x)$ for every $x$, it follows that for every $x$ of $T$, the point $(x, u(x))$ also lies on or below $\lambda$; that is,

$$
Q(u, \xi, x) \leqq k+\epsilon
$$

for every $x$ in $T$. Hence, if we denote the set

$$
E_{x}[Q(u, \xi, x)>k+\epsilon, x>\xi]
$$

by $E_{1}$, then $m_{e}\left(E_{1} I\right)<\eta l$. Since this holds for every $\eta>0$ however small, and for every $I$ of length $l<h$, we conclude that the exterior density of $E_{1}$ is zero at the point $\xi$. This shows that $A^{+}(u, \xi) \leqq k+\epsilon$, and since $\epsilon$ is arbitrary, it follows that

$$
A^{+}(u, \xi) \leqq k=A^{+}(f, \xi) \text {. }
$$

Therefore, by (2.1),

$$
A^{+}(u, \xi)=A^{+}(f, \xi) \text {. }
$$

Other parts of the lemma follow similarly.

3. Theorems. Theorem I states some properties of approximate derivatives which hold for both measurable and non-measurable functions. Some of these results are in the literature, but our proof is different and simpler. Theorem II gives sufficient conditions for the approximate derivability of arbitrary functions. Theorem III proves the principal result of this paper, namely the extension of Denjoy's theorem to approximate derivatives of arbitrary functions.

THEOREM I. If $f(x)$ is a finite function, then:

(i) The set of points for which the upper approximate derivative on one side is less than the lower approximate derivative on the other side is of measure zero.

(ii) If one of the approximate derivatives is finite at every point of a 
set $E$, it is equal to its opposite derivative at almost every point of $E$.

(iii) The set of points where the upper approximate derivative is $-\infty$, or the lower approximate derivative is $+\infty$, is of measure zero.

Proof. (i) It is sufficient to reason about the set

$$
E=E_{x}\left[A^{+}(x)<A_{-}(x)\right]
$$

since the corresponding set with the left and right sides interchanged may be treated similarly. If $x$ is a point of $E, A^{+}(x)$ cannot be $+\infty$. It follows that the set

$$
E_{x}[u(x)>f(x), x \in E]
$$

is of measure zero. For by the theorem of Blumberg, the point $(x, u(x))$ is fully approached by the curve $y=f(x)$ at almost every $x$; hence the inequality $u(x)>f(x)$ would imply, for almost every $x$ of $E$, that $A^{+}(x)=+\infty$. Accordingly, $u(x) \leqq f(x)$ for almost every point $x$ of $E$. But the set $E_{x}[u(x)<f(x)]$ is of measure zero. Consequently,

$$
u(x)=f(x)
$$

a.e. in $E$.

Now we apply Lemma 2, obtaining the equalities

$$
\begin{aligned}
& A^{+}(x)\left[=A^{+}(f, x)\right]=A^{+}(u, x), \\
& A_{-}(x)\left[=A_{-}(f, x)\right]=A_{-}(u, x)
\end{aligned}
$$

for almost every $x$ in $E$. Since $u(x)$ is measurable, the set $E_{x}\left[A^{+}(u, x)\right.$ $\left.<A_{-}(u, x)\right]$ is of measure zero-according to the results cited in the introduction for the approximate derivatives of measurable functions. Hence $E=E_{x}\left[A^{+}(x)<A_{-}(x)\right]$ is of measure zero.

(ii) Suppose $A^{+}(x)$ is finite at every point of a set $E$. As before, we must have

$$
u(x)=f(x) \quad \text { a.e. in } E
$$

and (3.1) applies. By the results for measurable functions just referred to, the measurable function $u(x)$ has a finite approximate derivative $A(u, x)$ a.e. on $E$. Hence

$$
A^{+}(u, x)=A_{-}(u, x) \quad \text { a.e. in } E .
$$

Consequently, by (3.1)

$$
A^{+}(x)=A_{-}(x) \quad \text { a.e. in } E \text {. }
$$

The reasoning for the cases where the other approximate derivatives are finite is similar.

(iii) It is sufficient to reason about the set 


$$
E=E_{x}\left[A^{+}(x)=-\infty\right]
$$

since the other cases are similar. By reasoning like that for (i), we have

$$
u(x) \leqq f(x) \quad \text { a.e. in } E .
$$

Since the set of the points for which $u(x)<f(x)$ is of measure zero, it follows that

$$
u(x)=f(x) \quad \text { a.e. in } E .
$$

We may consequently apply (3.1) and conclude that

$$
A^{+}(u, x)=A^{+}(x)=-\infty \quad \text { a.e. in } E \text {. }
$$

But for the measurable function $u(x)$, the set of points for which $A^{+}(u, x)=-\infty$ is of measure zero. Hence $E=E_{x}\left[A^{+}(x)=-\infty\right]$ is of measure zero.

THEOREM II. If $f(x)$ is a given finite function, let $E$ be the set of abscissas at which either the two approximate derivatives on the same side or the two upper (or lower) approximate derivatives are finite. Then $f(x)$ is approximately derivable at almost every point of $E$.

Proof. Let $E_{1}$ be the subset of $E$ at which $A^{+}(x)$ and $A_{+}(x)$ are finite. By Theorem I, case (i), we have

$$
A^{+}(x)=A_{-}(x), \quad A_{+}(x)=A^{-}(x) \quad \text { a.e. in } E_{1} .
$$

But since

$$
A^{+}(x) \geqq A_{+}(x), \quad A^{-}(x) \geqq A_{-}(x)
$$

for every $x$ in $E$, it follows that

$$
A^{+}(x)=A_{-}(x) \leqq A^{-}(x)=A_{+}(x) \quad \text { a.e. in } E_{1},
$$

whence $A^{+}(x)=A_{+}(x)$. The four approximate derivatives are consequently finite and equal, hence $f(x)$ is approximately derivable at almost every point of $E_{1}$. The other three possibilities may be treated similarly.

THEOREM III. For any finite function $f(x)$, the directional angle, in respect to approximate derivation, of the curve $y=f(x)$ is, at almost every $x$, either $0^{\circ}$ or $180^{\circ}$, or $360^{\circ}$.

Proof. The set of points $x$ for which any one of the relations

$$
f(x)>u(x), \quad f(x)<l(x),
$$




$$
u^{+}(x) \neq u^{-}(x), \quad l^{+}(x) \not l^{-}(x)
$$

holds is of measure zero (by the theorem of Blumberg and Lemma 1). Therefore we need only consider those points $x$ at which

$$
u^{+}(x)=u^{-}(x)=u(x), \quad l^{+}(x)=l^{-}(x)=l(x)
$$

and

$$
u(x) \geqq f(x) \geqq l(x) .
$$

Case (a). $u(x)>f(x)>l(x)$. From $u(x)>f(x)$, it follows that

$$
A^{+}(x)=+\infty, \quad A_{-}(x)=-\infty
$$

(cf. proof of Theorem I). Likewise, it follows from $f(x)>l(x)$ that

$$
A^{-}(x)=+\infty, \quad A_{+}\left({ }^{\prime} x\right)=-\infty
$$

Hence in this case the directional angle is $360^{\circ}$ almost everywhere on the set $E_{x}[u(x)>f(x)>l(x)]$.

Case (b). $u(x)=f(x)>l(x)$. (The relation $u(x)>f(x)=l(x)$ may be treated similarly.) From $f(x)>l(x)$, it follows, as before, that

$$
A^{-}(x)=+\infty, \quad A_{+}(x)=-\infty
$$

From $u(x)=f(x)$, it follows, by Lemma 2 , that

$$
A^{+}(x)=A^{+}(u, x), \quad A_{-}(x)=A_{-}(u, x) .
$$

If $A^{+}(u, x)$ is finite for almost every $x$ in a set $E$, then, on account of the measurability of $u(x), A^{+}(u, x)=A_{-}(u, x)$ almost everywhere in $E$. Hence $A^{+}(x)$ and $A_{-}(x)$ are finite and equal almost everywhere in $E$. The directional angle is, therefore, $180^{\circ}$ almost everywhere in $E$. If $A^{+}(u, x)=+\infty$ for almost every $x$ in $E$, then $A_{-}(u, x)=-\infty$ at almost every point of $E$ (again on account of the results for measurable functions). Hence $A^{+}(x)=+\infty$ and $A_{-}(x)=-\infty$ almost everywhere in $E$. Therefore the directional angle is $360^{\circ}$ almost everywhere in $E$.

Case (c). $u(x)=f(x)=l(x)$. By Lemma 2, we have at every point of $E=E_{x}[u(x)=f(x)=l(x)]$

$$
\begin{array}{ll}
A^{+}(x)=A^{+}(u, x), & A_{-}(x)=A_{-}(u, x), \\
A^{-}(x)=A^{-}(l, x), & A_{+}(x)=A_{+}(l, x) .
\end{array}
$$

Now since $u(x) \geqq l(x)$ for every $x$, it follows that

$$
\begin{array}{ll}
A^{+}(u, x) \geqq A^{+}(l, x), & A_{+}(u, x) \geqq A_{+}(l, x), \\
A^{-}(u, x) \leqq A^{-}(l, x), & A_{-}(u, x) \leqq A_{-}(l, x) .
\end{array}
$$


On the other hand, $u(x)$ and $l(x)$ are both measurable. The directional angle is, therefore, either $0^{\circ}$ or $360^{\circ}$ at the points of $y=u(x)$ and $y=l(x)$, for almost every $x$. Consequently,

$$
\begin{aligned}
& A^{+}(u, x)=A^{-}(u, x)=\sup A(u, x), \\
& A^{+}(l, x)=A^{-}(l, x)=\sup A(l, x), \\
& A_{+}(u, x)=A_{-}(u, x)=\inf A(u, x), \\
& A_{+}(l, x)=A_{-}(l, x)=\inf A(l, x),
\end{aligned}
$$

say, for almost every $x$. From (3.3) and (3.4) we obtain

$$
\sup A(u, x)=\sup A(l, x), \quad \inf A(u, x)=\inf A(l, x) \quad \text { a.e. in } E .
$$

Combining (3.2) and (3.5), we obtain, for almost every point $x$ of $E$.

$$
\begin{gathered}
A^{+}(x)=\sup A(u, x)=\sup A(l, x)=A^{-}(x), \\
A_{+}(x)=\inf A(u, x)=\inf A(l, x)=A_{-}(x) .
\end{gathered}
$$

Hence the directional angle of $y=f(x)$ is, almost everywhere in $E$, the same as that of $y=u(x)$ or $y=l(x)$; that is, either $0^{\circ}$ or $360^{\circ}$. This completes the proof.

REMARK. A measurable function $f(x)$ is aproximately continuous almost everywhere, and therefore

$$
u(x)=f(x)=l(x)
$$

Thus case (c) of Theorem III occurs almost everywhere, and the directional angle of $y=f(x)$ is, therefore, either $0^{\circ}$ or $360^{\circ}$ almost everywhere.

The following simple example shows that case $(\beta)$ (directional angle $=180^{\circ}$ ) may be realized for a non-measurable function on a set of positive measure. Let $I=E_{1}+E_{2}$ be a decomposition of the interval $I:(0,1)$ into two disjoint, non-measurable sets, each of exterior measure 1. Define $f(x)=1$ in $E_{1}, f(x)=0$ in $E_{2}$. It follows that case $(\beta)$ is valid at every point of $I$.

The results of this paper on approximate derivatives, in particular Theorems I, II and III, are readily extensible to the case where $x$ ranges over an arbitrary set instead of an interval. They are also easily extended to many-valued functions.

\section{BIBLIOGRAPHY}

1. S. Saks, (1) Sur les nombres dérivés des fonctions, Fund. Math. vol. 5 (1924) pp. 98-104. (2) Theory of integrals, 1937, pp. 295-297.

2. E. H. Hanson, $A$ theorem of Denjoy, Young and Saks, Bull. Amer. Math. Soc. vol. 40 (1934) pp. 691-694. 
3. H. Blumberg, The measurable boundaries of an arbitrary function, Acta Math. vol. 65 (1935) pp. 263-282.

4. J. C. Burkill and U. S. Haslam-Jones, (1) The derivatives and approximate derivatives of measurable functions, Proc. London Math. Soc. (2) vol. 32 (1931) pp. 346-355. (2) Relative measurability and the derivatives of non-measurable functions, Quart. J. Math. Oxford Ser. vol. 4 (1933) pp. 233-239.

5. A. J. Ward, On the points where $A D_{+}>A D^{-}$, J. London Math. Soc. vol. 8 (1933) pp. 295-299.

6. R. L. Jeffery, The derivatives of arbitrary functions over arbitrary sets, Ann. of Math. vol. 36 (1935) pp. 438-447.

7. S. Saks, Review of [6], Zentralblatt für Mathematik vol. 11 (1935) p. 341.

Ohio State University and

Central University, Nanking, China 